Cite this: J. Mater. Chem. C, 2014, 2 , 2423

Received 12th December 2013 Accepted 11th January 2014

DOI: $10.1039 /$ c3tc32465f

www.rsc.org/MaterialsC

\section{Spray-coatable ionogels based on silane-ionic liquids for low voltage, flexible, electrolyte-gated organic transistors $\uparrow$}

\author{
S. Thiemann, ${ }^{a}$ S. J. Sachnov, ${ }^{b}$ M. Gruber, ${ }^{a}$ F. Gannott, ${ }^{a}$ S. Spallek, ${ }^{c}$ M. Schweiger, ${ }^{a}$ \\ J. Krückel, ${ }^{a}$ J. Kaschta, ${ }^{a}$ E. Spiecker, ${ }^{c}$ P. Wasserscheid ${ }^{b}$ and J. Zaumseil ${ }^{\star a}$
}

We introduce a new type of silane-based ionogels that are produced by gelation of the ionic liquid 3-methyl-1-(3-(triethoxysilyl)propyl)-imidazolium bis(trifluoromethylsulfonyl)imide ([(EtO) 3 SiPMIM][TFSI]) with tetramethylorthosilane and formic acid. In the obtained ionogels the cations are involved in the network formation while the anions can move freely. The ionogels show advantageous properties for application in flexible electronics, such as low modulus, solution processability and high specific capacitance. Spray-coated ionogels were used as high capacitance gate dielectrics for organic (poly[3hexylthiophene], $\mathrm{P} 3 \mathrm{HT}$ ) electrolyte-gated transistors (EGTs) that operated at very low voltages ( $<2 \mathrm{~V})$ with high on/off ratios in air over weeks. Devices fabricated on polymer foil remained functional during repeated bending cycles with strains up to $2.3 \%$.

\section{Introduction}

Ionogels (or iongels) are a class of hybrid materials that combine the properties of ionic liquids (ILs, molten organic salts with a melting temperature below $100{ }^{\circ} \mathrm{C}$ ) with those of another component (inorganic or organic) in order to obtain a solid and malleable material while retaining the specific properties of the IL, especially a large electrochemical window, high specific capacitance, and high ionic conductivity. They are produced by mixing the ionic liquid with a matrix that forms a solid host network. Ionogels are applicable as electrolytes in batteries, ${ }^{1}$ supercapacitors, ${ }^{2-5}$ dye-sensitized solar cells (DSSCs), ${ }^{6-8}$ and electrolyte-gated organic ${ }^{9-11}$ or inorganic ${ }^{12-16}$ transistors. With this wide range of possible applications in mind interest in these new materials has increased substantially over the last decade. Le Bideau et al. recently reviewed the most promising ways to generate ionogels without losing the advantageous properties of the ionic liquid. ${ }^{17}$ One method is to add small amounts of a triblock-copolymer with soluble and insoluble segments to an ionic liquid. The swollen copolymer

\footnotetext{
${ }^{a}$ Institute of Polymer Materials, Friedrich-Alexander-Universität Erlangen-Nürnberg, Martensstraße 7, D-91058 Erlangen, Germany. E-mail: jana.zaumseil@fau.de

${ }^{b}$ Department of Chemical and Bioengineering, Friedrich-Alexander-Universität Erlangen-Nürnberg, Egerlandstraße 3, D-91058 Erlangen, Germany

${ }^{c}$ Center for Nanoanalysis and Electron Microscopy, Friedrich-Alexander-Universität Erlangen-Nürnberg, Cauerstraße 6, 91058 Erlangen, Germany

$\dagger$ Electronic supplementary information (ESI) available: Differential scanning calorimetry (DSC) and thermal gravimetric analysis (TGA) of the silane precursor, displacement current characteristics of P3HT-EGTs and calculated capacitance vs. gate voltage, bias stress stability of P3HT-EGTs in nitrogen. See DOI: $10.1039 / \mathrm{c} 3 \mathrm{tc} 32465 \mathrm{f}$
}

forms a network, in which both cations and anions are still mobile. ${ }^{18}$ These ionogels are often used as electrolytes in electrolyte-gated transistors (EGTs). EGTs with triblock-copolymer ionogels are promising components for printable and flexible electronics ${ }^{\mathbf{1 0 , 1 9 , 2 0}}$ as they are processed from solution at low temperatures and allow for efficient transistor operation at very low applied voltages.

In an EGT the gate dielectric is replaced by an electrolyte (e.g., an ionogel). When a gate voltage is applied, the anions and cations move toward the gate electrode and the semiconductor, respectively. There they form nm-thick electric double layers (EDLs) at the interfaces with large effective capacitances in the $\mu \mathrm{F} \mathrm{cm}^{-2}$ range, which enable the accumulation of large charge carrier densities at low voltages. In the case of polymer semiconductors the ions penetrate into the bulk so that opposite charges are accumulated to compensate. ${ }^{20,21}$ In both cases the carrier density and thus conductivity of the channel vary with the applied gate voltage as in a typical field-effect transistor. Given that ions within the ionogel retain their high mobility good switching speeds are attainable. ${ }^{12,14}$

Besides triblock-copolymer ionogels, gelation of ionic liquids is also achievable by mixing the ILs with other synthetic polymers (e.g., poly[vinylidenefluoride-co-hexafluoro-propylene]), ${ }^{22}$ biopolymers, such as cellulose, ${ }^{23}$ or silicon dioxide nanoparticles, ${ }^{24}$ to name a few. Furthermore, ionogels can be obtained by forming silica-like networks via a sol-gel process in the presence of ionic liquids. Néouze et al. investigated the properties of such ionogels based on silicon alkoxide precursors like tetramethylorthosilane (TMOS) and tetraethylorthosilane (TEOS), formic acid (FA) and different ionic liquids, e.g., 1-butyl-3-methylimidazolium bis(trifluoromethylsulfonyl)imide ([BMIM][TFSI]). ${ }^{25-29}$ The precursors 
crosslink via a condensation reaction and form an open silica-like network, through which the anions and cations can percolate. Depending on the amount and type of the IL, hard, transparent, and insoluble ionogels can be formed with high ionic conductivities and good thermal stability. Horowitz and Panzer optimized the TMOS : FA : IL ratio to obtain a compliant gel with high ionic conductivity and high capacitance, comparable to the pure IL. ${ }^{30}$ Importantly, in these ionogels the ionic liquid is not involved in the network formation itself but simply fills the voids and all ions remain free to move.

Here we introduce an ionogel, in which the ionic liquid itself takes part in the network formation. We use an ionic liquid with silicon alkoxide (triethoxysilane) functional groups attached to the imidazolium cation. This type of ionic liquid was previously used to immobilize palladium complexes on silica particles for heterogeneous catalysis. ${ }^{31}$ Without any additional ILs we produce ionogels by gelation with TMOS and FA that are viscous but mechanically stable. The new silane-based ionogels are soluble in acetone and ethylacetate thus enabling solution processing, e.g., printing and spray-coating, at low temperatures. We successfully apply these ionogels in low-voltage electrolyte-gated polymer transistors and simple circuits on rigid and flexible substrates.

\section{Experimental}

\section{Ionogel synthesis}

The ionic liquid (3-methyl-1-(3-(triethoxysilyl)propyl)-imidazolium bis(trifluoromethylsulfonyl)imide, $\left.\quad\left[(\mathrm{EtO})_{3} \mathrm{SiPMIM}\right][\mathrm{TFSI}]\right)$ was prepared from 1-methyl-3-(3-trimethoxysilylpropyl)imidazolium chloride, which was synthesized according to Lee et $a .^{31}$ Subsequently the chloride was substituted with the [TFSI] anion by anion exchange. ${ }^{32,33}$ The IL was washed three times and dried over $\mathrm{MgSO}_{4}$. For the preparation of the ionogel $\left[(\text { EtO })_{3}\right.$ SiPMIM $][$ TFSI $]$ was mixed with formic acid (FA) and tetramethylorthosilane (TMOS) in an optimized molar ratio of FA : TMOS : $\left[(\text { EtO })_{3}\right.$ SiPMIM $][$ TFSI $]=61: 1: 3$. The mixture was placed on a hotplate at $150{ }^{\circ} \mathrm{C}$ for 12 hours. Differential scanning calorimetry and thermal gravimetric analysis of the precursor mainly show the evaporation of formic acid, ethylformate and methylformate starting at $44{ }^{\circ} \mathrm{C}$. No further mass loss or decomposition are observed up to $400{ }^{\circ} \mathrm{C}$ (see Fig. S1, ESI $\dagger$ ). After cooling to room temperature a highly viscous, sticky ionogel was obtained (see Fig. 1). The ionogel was soluble in acetone and ethylacetate after $20 \mathrm{~min}$ of ultrasonication at room temperature. For further processing a solution in acetone with a concentration of $360 \mathrm{mg} \mathrm{ml}^{-1}$ was prepared. All processing steps were carried out in ambient air.

\section{Ionogel characterization}

Electrochemical impedance spectroscopy (EIS) and cyclic voltammetry (CV) measurements of the pure ionic liquid were performed with an Autolab potentiostat PGSTAT30 (Metrohm) in a closed sample container in air. The IL was placed in a sealable electrochemical cell (RHD instruments), which accommodated two- and three-electrode configurations. In the two-electrode setup (e.g. for EIS measurements) the platinum sample container (volume $<1.6 \mathrm{ml}$ ) acted as the counter electrode and a polished glass-sealed platinum-wire (diameter $0.25 \mathrm{~mm}$ ) served as the working electrode. When a three-electrode setup was required, e.g. in the case of $\mathrm{CV}$ measurements, an additional glass-sealed platinum wire was used as a pseudoreference electrode. The sweep rate for all $\mathrm{CV}$ measurements was $50 \mathrm{mV} \mathrm{s}^{-1}$ and the temperature was $20{ }^{\circ} \mathrm{C}$. EIS measurements of the ionogel were performed with a Novocontrol modular measurement system consisting of an Alpha-AK high resolution impedance analyser and a POT/GAL15V/10A electrochemical interface. In a two-electrode setup for solid and gel-like samples the ionogels were sandwiched between two Au-plated stainless steel electrodes. Impedance spectra were recorded for frequencies from $1 \mathrm{MHz}$ to $0.1 \mathrm{~Hz}$ with an oscillation amplitude of $10 \mathrm{mV}$ and a dc-bias of $0 \mathrm{~V}$ at $20^{\circ} \mathrm{C}$.

The morphology of the ionogels (spray-coated onto TEMgrids) was studied by high resolution transmission electron microscopy (HRTEM, Philips CM300). The film thickness of the spray-coated ionogel was measured by laser profilometry with a UBM microfocus system. Raman spectra were acquired using a Renishaw inVia Reflex Confocal Raman Spectrometer with an excitation laser wavelength of $785 \mathrm{~nm}$. Precursor solutions were kept in a sealed glass cuvette and spray-coated ionogels and pure ionic liquid were deposited onto aluminum foil for background-free measurements.
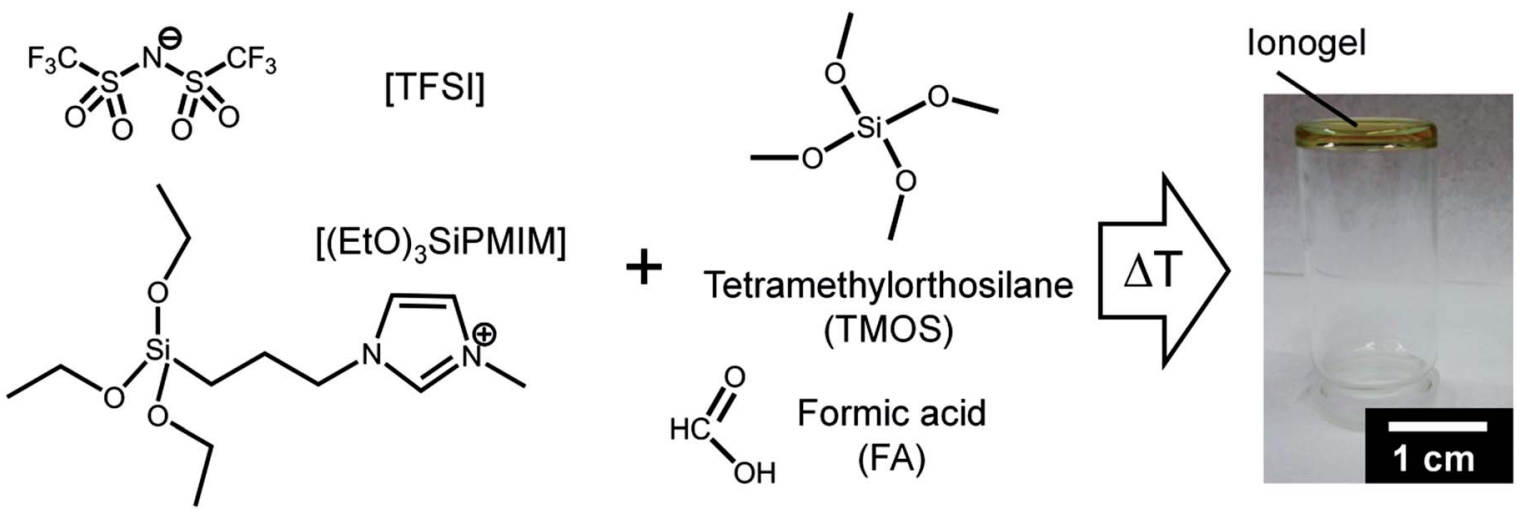

Fig. 1 Molecular structures of the ionic liquid [(EtO) $\left.{ }_{3} \mathrm{SiPMIM}\right][T F S I]$ and network precursors with optical image of the resulting ionogel. 
Differential scanning calorimetry (DSC) and thermal gravimetric analysis (TGA) were performed with a Q 2000 differential scanning calorimeter and a Q5000 IR thermo-gravimetric analyzer (TA Instruments), respectively.

Oscillatory shear measurements were performed on a Gemini rotational rheometer (Malvern) in the plate-plate geometry with a diameter of $25 \mathrm{~mm}$ and a gap of $1.8 \mathrm{~mm}$. The dynamic shear moduli and the viscosity were recorded as a function of the angular frequency in the linear viscoelastic regime. The temperature was $25{ }^{\circ} \mathrm{C}$ and the stress amplitude was $200 \mathrm{~Pa}$ in the small amplitude oscillatory shear (SAOS) mode.

\section{Device fabrication and characterization}

Regio-regular poly(3-hexylthiophene) (P3HT, Plextronics, Plexcore ${ }^{\circledR}$ OS $\left.2100, M_{\mathrm{w}}=54000-75000 \mathrm{~g} \mathrm{~mol}^{-1}\right)$ was spincoated from 1,2-dichlorobenzene $\left(3 \mathrm{mg} \mathrm{ml}^{-1}\right)$ on glass substrates (Schott AF32 Eco) or polyethyleneterephthalate foil (PET, Mitsubishi Polyester film, thickness $50 \mu \mathrm{m}$ ) with photolithographically pre-patterned chromium/gold $(2 \mathrm{~nm} / 30 \mathrm{~nm})$ interdigitated source-drain electrodes (channel width $W=20$ $\mathrm{mm}$, channel length $L=20 \mu \mathrm{m}$ ) in nitrogen atmosphere and annealed for $30 \mathrm{~min}$ at $150{ }^{\circ} \mathrm{C}$.

Smooth, homogeneous films of the ionogel with approximately $100 \mu \mathrm{m}( \pm 20 \mu \mathrm{m})$ thickness were obtained by spraying the ionogel-acetone solution with an air-brush gun twice for roughly $15 \mathrm{~s}$ at a distance of 10 to $15 \mathrm{~cm}$ onto the substrates at $60{ }^{\circ} \mathrm{C}$ in ambient air. A thin polydimethylsiloxane film (PDMS, Corning Sylgard 184, thickness $450 \mu \mathrm{m}$ ) with rectangular openings acted as a simple shadow mask. Samples were stored in a vacuum at $40{ }^{\circ} \mathrm{C}$ for 12 hours to remove residual solvent and air-bubbles from the spray-coated ionogel. Finally, a gold gate electrode was evaporated onto the ionogel by electron-beam evaporation (50 nm, $0.05 \mathrm{~nm} \mathrm{~s}^{-1}$ ).
Current-voltage characteristics of ionogel-gated P3HT transistors and inverters were measured with an Agilent 4155C parameter analyzer in a dry nitrogen glovebox, and in ambient air where noted. Resistor-loaded inverters were realized with an external resistor $(2 \mathrm{M} \Omega)$.

\section{Results and discussion}

\section{Ionogel properties}

For the synthesis of the ionogel an optimised molar ratio of FA : TMOS : [(EtO) ${ }_{3}$ SiPMIM] [TFSI $]=61: 1: 3$ was found (see Fig. 1). The reaction gave a viscous, sticky ionogel with a capacitance of $10.4 \mu \mathrm{F} \mathrm{cm} \mathrm{cm}^{-2}$ (determined from EIS measurements). The stiffness and capacitance of the ionogel were tunable by the TMOS precursor content. Hard ionogels were obtained by using a larger amount of TMOS. Much softer, more liquid gels resulted from a tenth of the TMOS content. Those showed higher capacitances of $18 \mu \mathrm{F} \mathrm{cm} \mathrm{cm}^{-2}$, probably because fewer $\left[(\mathrm{EtO})_{3} \mathrm{SiPMIM}\right]$ cations were involved in the network formation. This observation is in agreement with results by Horowitz and Panzer. ${ }^{30}$ The sticky, honey-like ionogel was mechanically stable after spray-coating or lamination for months. Ionogels with this precursor ratio gave the best and most homogenous films after spray-coating. Adding a small amount of a second IL, such as [EMIM][TFSI] (1-ethyl-3-methylimidazolium bis(trifluoromethylsulfonylimide)), increased the capacitance but reduced the mechanical stiffness. As previously described by Néouze et al. for silica-derived networks the amount and nature of the IL directly influence the properties of the ionogel. ${ }^{26}$

In order to verify the crosslinking and formation of a network, we recorded Raman spectra of the pure IL [(EtO $)_{3}$ SiPMIM][TFSI], the precursor solution (IL : TMOS : FA) and the spray-coated ionogel. In Fig. 2a the spectral regions between (a)

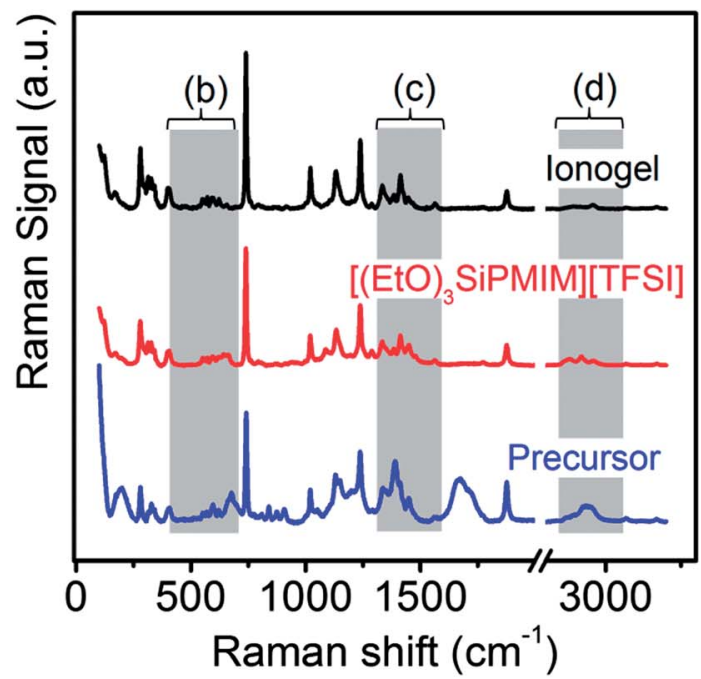

(b)

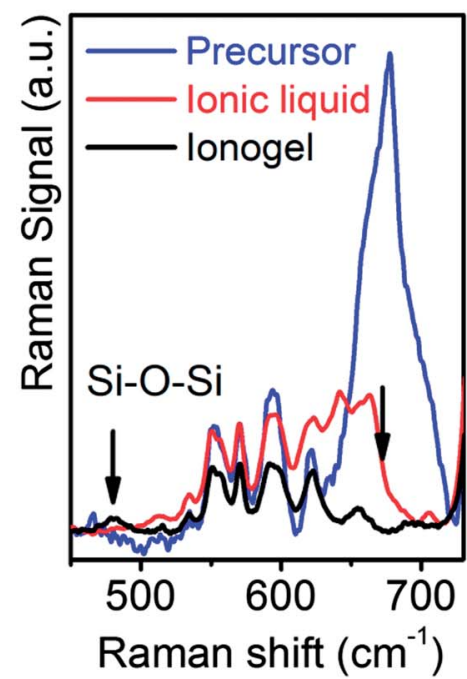

(c)

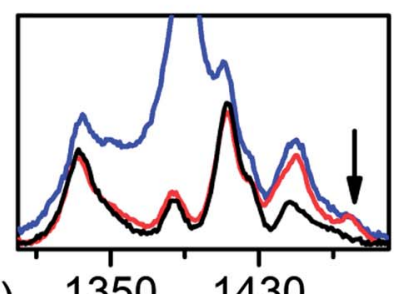

(d)

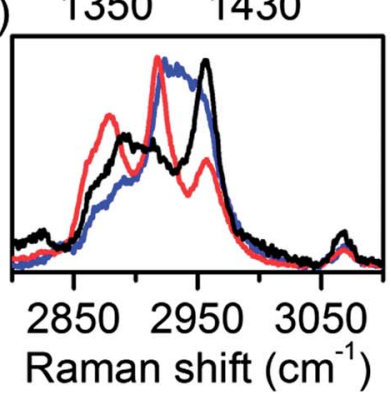

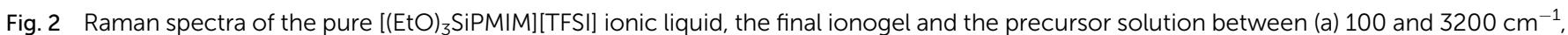
(b) 450 and $710 \mathrm{~cm}^{-1}$, (c) 1300 and $1500 \mathrm{~cm}^{-1}$, and (d) 2800 and $3100 \mathrm{~cm}^{-1}$. 
$100-2000 \mathrm{~cm}^{-1}$ and $2800-3200 \mathrm{~cm}^{-1}$ are shown, and Fig. $2 \mathrm{~b}-\mathrm{d}$ show the most important peaks in detail. Five peaks at 739, $1020,1132,1238$, and $1877 \mathrm{~cm}^{-1}$ are dominant in all three samples. These arise from the characteristic vibrations of the [TFSI] anion and imidazolium cation and do not change after gelation. ${ }^{34,35}$ More informative are those peaks that increase or decrease after gelation. A small peak at $490 \mathrm{~cm}^{-1}$ emerges after gelation. This Raman mode is representative of Si-O-Si bonds corroborating at least partial formation of a silica-like network. A peak at $665 \mathrm{~cm}^{-1}$ corresponding to a Si-O-C p.h. (p.h. partially hydrated) stretching mode and two peaks at 1448 and $1476 \mathrm{~cm}^{-1}$ corresponding to asymmetric and symmetric bending modes of $-\mathrm{CH}_{3}$, respectively, ${ }^{34}$ decrease substantially. From these spectra and in accordance with Martinelli and Nordstierna ${ }^{34}$ we can summarize the sol-gel reaction as follows: the formic acid hydrolyzes the alkoxy silane groups of the IL and the TMOS. Ethylformate and methylformate are most likely formed and evaporate immediately at the reaction temperature of $150{ }^{\circ} \mathrm{C}$ as indicated by the reduction of the $\mathrm{Si}-\mathrm{O}-\mathrm{C}_{\text {p.h. peaks }}$ and the mass loss found in the TGA. The silanol groups then undergo a condensation reaction creating a $\mathrm{Si}-\mathrm{O}-\mathrm{Si}$ network (indicated by the peak at $490 \mathrm{~cm}^{-1}$ ) containing the cations of the ionic liquid and silica originating from the TMOS. Although the Raman signals of the $\mathrm{Si}-\mathrm{O}-\mathrm{C}_{\mathrm{p} . h}$. and $-\mathrm{CH}_{3}$ groups are strongly reduced in the final ionogel they have not completely vanished indicating free network ends and remaining free $\left[(\mathrm{EtO})_{3} \mathrm{SiPMIM}\right]$ cations. We conclude that the ionogel is partially crosslinked by Si-O-Si bonds, with the cations of the IL involved in the network. The softness (see rheology data below) of the obtained ionogel suggests that this is not a fully formed, three-dimensional network as those demonstrated by Néouze et $a .^{25-27}$ but rather a highly branched polyelectrolyte or ionomer with fixed cations and mobile anions. The lack of complete crosslinking and the presence of positive charges within the network would also explain the solubility of the ionogel in polar solvents such as acetone and ethylacetate after sonication. Polyelectrolytes based on, for example, poly(styrene sulfonic acid) and others have been previously used for EGTs. ${ }^{36-38}$

We investigated the rheological properties of the obtained ionogel by means of oscillatory shear measurements. Fig. 3a shows the storage modulus $\left(G^{\prime}\right)$ and the loss modulus $\left(G^{\prime \prime}\right)$ as well as the absolute value of the complex viscosity $\left(\left|\eta^{*}\right|\right)$ as a function of the angular frequency $(\omega)$. Over the investigated frequency range $G^{\prime \prime}$ is proportional to $\omega$ and exceeds $G^{\prime}$. This behaviour is typical of a liquid. $G^{\prime}$ exhibits a plateau between 0.1 and $1 \mathrm{rad} \mathrm{s}^{-1}$ indicating a second relaxation process. According to Capek $^{39}$ ionic polymers frequently show microphase separation leading to ion-rich domains due to strong ion-dipole attractions. Due to the formation of a second phase and surface energy differences between the phases an additional relaxation process occurs at low frequencies or elevated temperatures. At frequencies below and above the plateau $G^{\prime}$ scales roughly with $\omega^{2}$ indicating typical terminal rheological behaviour of a viscoelastic liquid. ${ }^{\mathbf{4 0}}$ The rheological data show that for the low TMOS concentrations used here the produced ionogel behaves more like a highly viscous liquid than a gel. This indicates again (a)

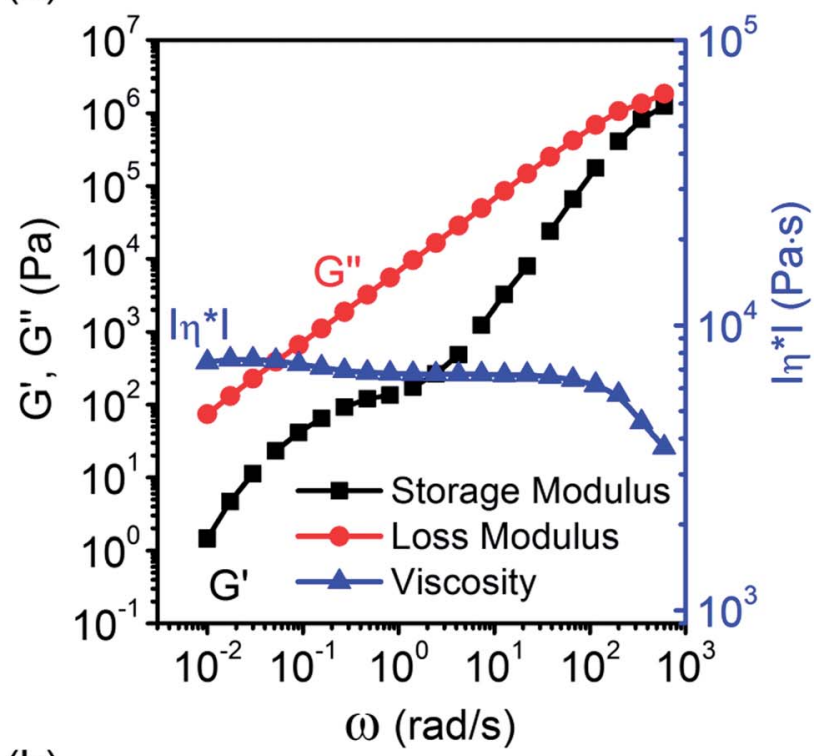

(b)

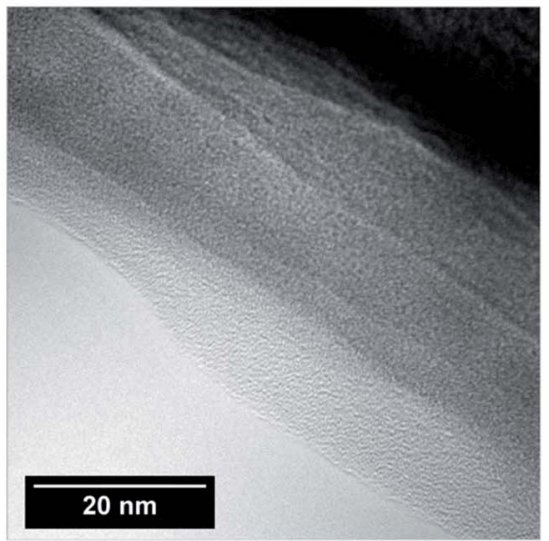

Fig. 3 (a) Loss $\left(G^{\prime \prime}\right)$ modulus, storage $\left(G^{\prime}\right)$ modulus and viscosity $\left(\left|\eta^{*}\right|\right)$ versus angular frequency $(\omega)$ in the viscoelastic regime for a silanebased ionogel. (b) HRTEM image of the spray-coated ionogel on a thin carbon membrane.

that crosslinking is incomplete and branched ionomers or polyelectrolytes are formed instead of a complete network.

HRTEM images (see Fig. 3b) as well as electron diffraction patterns (not shown) indicate an amorphous morphology. There is no indication for the presence of any larger silica structures.

For the application of ionogels as high capacitance electrolytes their electrochemical properties are crucial. The electrochemical window of the pure $\left[(\mathrm{EtO})_{3}\right.$ SiPMIM $][$ TFSI $]$ extends from $-1.5 \mathrm{~V}$ to $2.0 \mathrm{~V}$. The ionogel exhibits the same overall electrochemical window of $3.5 \mathrm{~V}$ but slightly shifted (see Fig. 4a). Electrochemical impedance spectroscopy (EIS) was carried out to obtain phase angle versus frequency $(f)$ plots for the ionogel and the pure IL. In typical electrolyte systems small phase angles at high frequencies are indicative of resistor-like behaviour and phase angles close to $-90^{\circ}$ are representative of capacitive behaviour. ${ }^{41}$ The pure IL shows an almost constant phase angle $\left(\max .-83^{\circ}\right)$ at frequencies up to $100 \mathrm{~Hz}$, which 
(a)

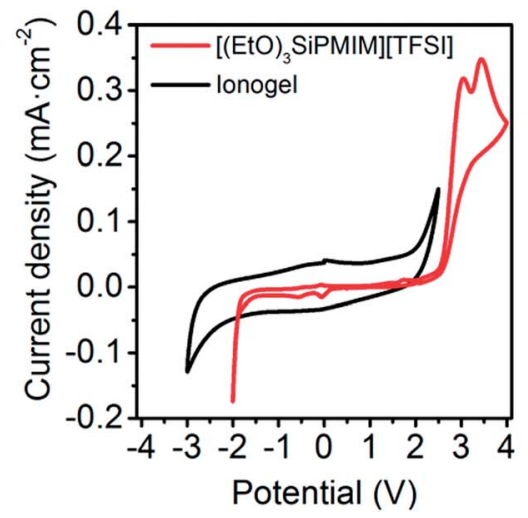

(b)

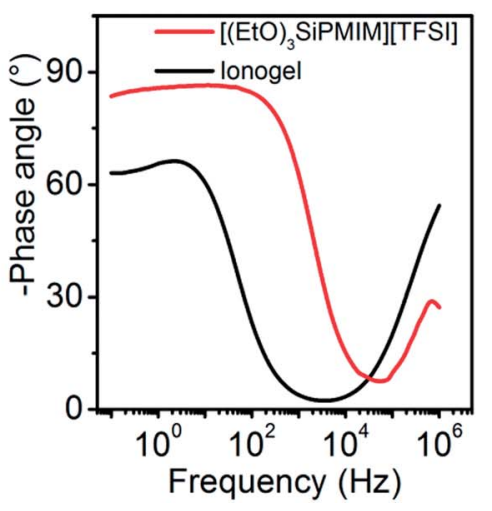

(c)

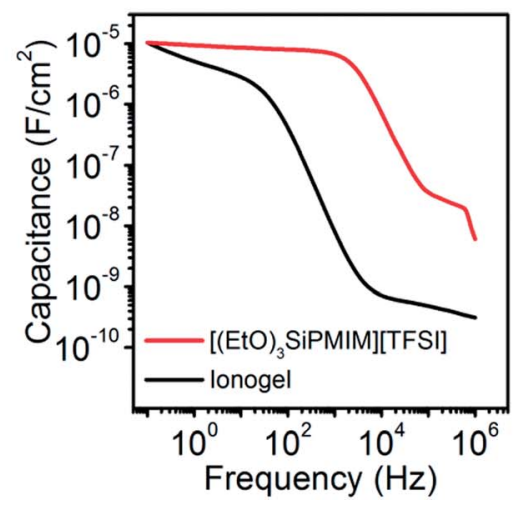

Fig. 4 (a) Cyclic voltammograms of [(EtO) ${ }_{3}$ SiPMIM][TFSI] and spray-coated ionogels. Frequency-dependent phase angle (b) and calculated specific capacitance (c) of the ionic liquid and ionogel (measured in ambient air).

drops to $0^{\circ}$ at $100 \mathrm{kHz}$. In contrast to that, the ionogel shows a maximum phase angle of $-66^{\circ}$ and starts to drop at frequencies larger than $10 \mathrm{~Hz}$. At much higher frequencies $\left(>10^{4} \mathrm{~Hz}\right)$ the phase angle rises again up to $54^{\circ}$, possibly due to dipolar relaxation of the material, as previously shown by Larsson et al. for polyelectrolytes. ${ }^{37}$ The frequency-dependent capacitances of the ionogel and IL (see Fig. 4c) were calculated according to Dasgupta et $a l .{ }^{42}$ Note that, a serial model of a resistor and a constant phase element was used to extract the capacitances. The IL and the ionogel exhibit similar specific capacitances of $10.4 \mu \mathrm{F} \mathrm{cm}^{-2}$ at the lowest frequency of $0.1 \mathrm{~Hz}$. The decrease of capacitance of the ionogel around $100 \mathrm{~Hz}$ indicates a relatively low ionic mobility within the ionogel, which is in agreement with the measured ionic conductivity of $14.5 \mu \mathrm{S} \mathrm{cm}^{-1}$ compared to the pure IL ( $\left.900 \mu \mathrm{S} \mathrm{cm}^{-1}\right)$. The resulting RC-time constant of $740 \mu \mathrm{s}$ for an ionogel thickness of $100 \mu \mathrm{m}$ would result in a relatively low switching speed for transistors gated with these ionogels. Clearly, the involvement of the cation in the network and its interaction with the anions slow down the ion movement and thus the formation of electric double-layers. Thinner ionogel films, e.g., produced by printing, would improve the switching speed as previously shown for other ionogels. ${ }^{41}$

Despite the partial crosslinking the obtained ionogels are soluble in common organic solvents like acetone and thus solution processable, e.g., by spray-coating at low temperatures, which enables the use of low-cost, flexible polymer substrates. Also, these new ionogels allow for the evaporation of thin metal films on top as gate electrodes (see the inset in Fig. 5b). This is unusual because most metals penetrate common ionogels used for EGTs during evaporation and do not form continuous or conducting films.

\section{Ionogel-gated P3HT transistors and circuits}

In order to test whether the new silane-IL based ionogels can act as high capacitance gate dielectrics we fabricated FETs with interdigitated gold electrodes and spin-coated P3HT as the semiconducting layer on a glass substrate. P3HT is a typical hole conductor and the free [TFSI] anions will penetrate the polymer layer when a negative gate voltage is applied. The (a)

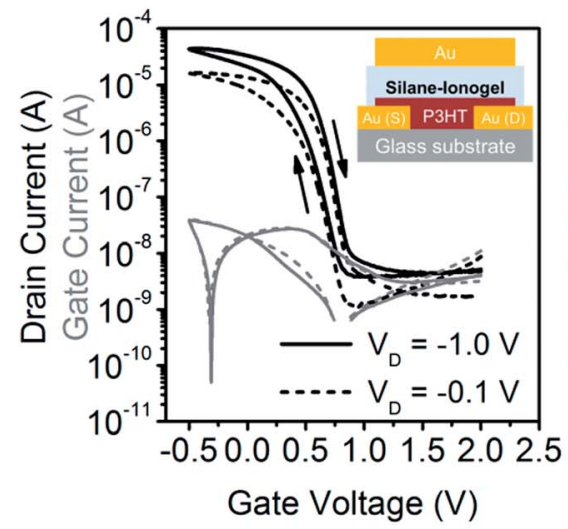

(b)

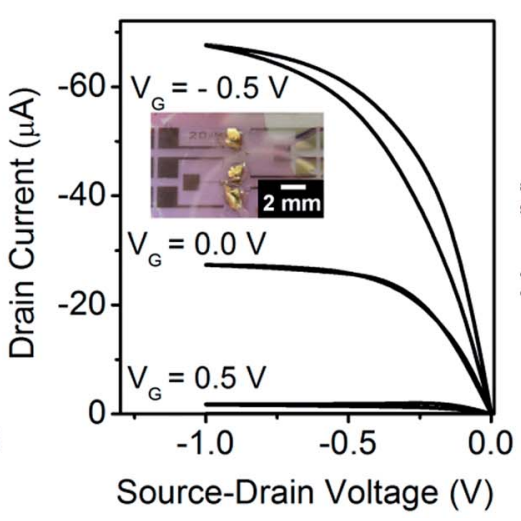

(c)

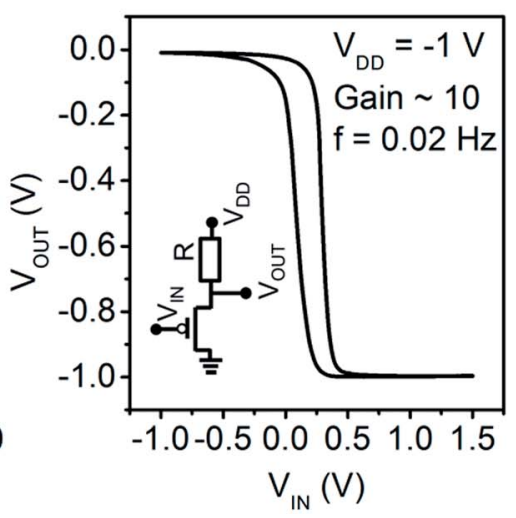

Fig. 5 Transfer characteristics of the ionogel-gated P3HT transistor (sweep rate $80 \mathrm{mV} \mathrm{s}^{-1}$ ); inset: device geometry (S - source, D - drain). (b) Output characteristics, inset: optical image of the device with evaporated gold gate electrodes. (c) Transfer curve of the resistor-loaded inverter with the ionogel-gated P3HT transistor, inset: circuit diagram. 
transfer and output characteristics of these ionogel-gated P3HTtransistors measured in dry nitrogen are shown in Fig. $5 a$ and b. A schematic illustration of the device geometry and an optical micrograph are shown in the insets. All EGTs operated at very low gate voltages between $2.0 \mathrm{~V}$ and $-1.0 \mathrm{~V}$ with on/off ratios of $10^{3}-10^{4}$. The gate leakage currents ranged from 1 to $10 \mathrm{nA}$. By patterning the semiconductor and the dielectric (e.g., by printing) in order to minimize unnecessary overlap with the gate electrode the leakage- and off-currents could be reduced further. The devices were measured at a voltage sweep rate of $80 \mathrm{mV} \mathrm{s}^{-1}$ exhibiting moderate current hysteresis probably due to the slow ionic movement within the ionogel and necessary penetration of the polymer layer. This hysteresis can be decreased further by using a lower sweep rate. The positive turnon voltages $V_{\mathrm{ON}}(1.2 \mathrm{~V} \pm 0.5 \mathrm{~V})$ and threshold voltages $V_{\mathrm{TH}}(0.9 \mathrm{~V}$ $\pm 0.4 \mathrm{~V}$ ) confirm the expected p-doping of the semiconductor due to the ionogel deposition in ambient air. Exposure to air usually leads to large positive turn-on voltages in P3HT transistors and increases the off-currents substantially by oxygen doping. ${ }^{43}$ This effect is suppressed here, due to the efficient three-dimensional depletion of holes at positive gate voltages in EGTs. All ionogel-gated P3HT transistors exhibit typical output characteristics with a linear current increase at small sourcedrain voltages $\left(-V_{\mathrm{D}}<-0.2 \mathrm{~V}\right)$ and current saturation at higher negative source-drain voltages. Linear and saturation fieldeffect mobilities were calculated according to standard equations for ideal field-effect transistors. The extracted saturation field-effect mobilities for holes in P3HT were relatively low with $0.024 \mathrm{~cm}^{2} \mathrm{~V}^{-1} \mathrm{~s}^{-1}$. The calculated linear mobilities were slightly higher with $0.037 \mathrm{~cm}^{2} \mathrm{~V}^{-1} \mathrm{~s}^{-1}$. Note that, the deposition conditions of P3HT (spincoated from 1,2-dichlorobenzene) were not optimized to obtain the ideal microstructure of P3HT for fast charge transport.

In order to calculate the field-effect mobilities the specific capacitance $C_{\mathrm{i}}$ of the ionogel was required. The formation of the EDL and therefore also the effective capacitance of the ionogel depend on the applied gate voltage and the nature of the interface and penetration of ions into the polymer. Hence, the capacitance was measured in the actual device instead of using the quasi-static values from EIS obtained at zero bias and with two gold electrodes. Displacement current measurements were carried out to extract the gate voltage dependent capacitance according to Xie and Frisbie. ${ }^{44}$ The source and drain electrodes were grounded and the displacement current $\left(I_{\text {Displ }}\right)$ (i.e. the gate current) was measured for different gate voltage sweep rates $\left(\mathrm{d} V_{\mathrm{G}} / \mathrm{d} t\right)$ (see Fig. S2, ESI $\dagger$ ). The extracted capacitance values increased from $0.6 \mu \mathrm{F} \mathrm{cm}^{-2}$ at $0.6 \mathrm{~V}$ to $3.2 \mu \mathrm{F} \mathrm{cm}{ }^{-2}$ at $-0.5 \mathrm{~V}$. The capacitance values obtained by EIS (see above) and displacement current measurements differ considerably, probably due to the fact that $\mathrm{P} 3 \mathrm{HT}$ is permeable for the [TFSI] anions leading to electrochemical doping instead of the formation of electric double layers on the metal electrodes used for EIS. ${ }^{20}$ For the mobility calculations above the capacitances extracted from displacement current measurements were used.

In order to test the applicability of these ionogel-gated transistors in simple circuits we fabricated a resistor loaded inverter consisting of a P3HT EGT and an external resistor
( $2 \mathrm{M} \Omega$ ). The circuit diagram and the obtained transfer curve are shown in Fig. 5c. The hysteresis is probably a result of the limited ionic conductivity and thickness of the ionogel. The operating frequency was quite low with $0.02 \mathrm{~Hz}$ but the gain of 10 was reasonable. At higher operating frequencies the hysteresis increased and the gain was reduced. The switching time of the inverter would decrease for thinner ionogels and shorter channel lengths.

We tested the device performance of the P3HT EGTs not only in nitrogen atmosphere but also in air. For application in flexible electronics the long-term stability in both atmospheres is essential. The stability of the ionogel-gated P3HT EGTs was first tested in dry nitrogen by repeated transfer measurements every $5 \mathrm{~min}$ for about 13 hours. The on/off current ratio $\left(\sim 4 \times 10^{4}\right)$ did not change over time but the corresponding transfer curves showed a negative threshold shift of about $0.25 \mathrm{~V}$ (see Fig. S3, ESI $\dagger$ ).

For measurements in air we expected a strong decrease of the on/off ratio and a threshold voltage shift to positive voltages due to the increased doping of P3HT by oxygen, which is usually observed for P3HT transistors in air and light. ${ }^{43,45}$ However, the

(a)

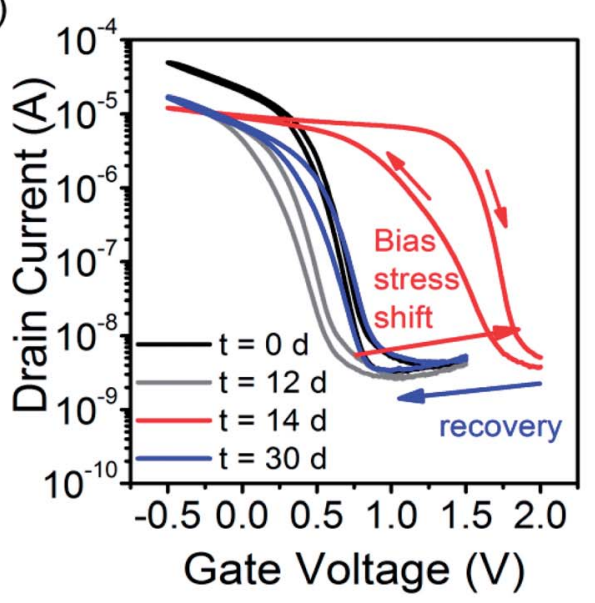

(b)

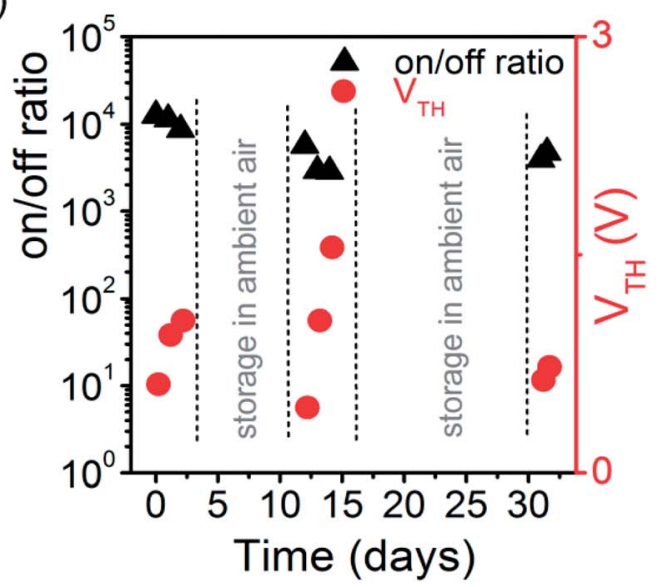

Fig. 6 (a) Transfer characteristics $\left(V_{D}=-1.0 \mathrm{~V}\right)$ of the ionogel-gated P3HT transistor after $0,12,14$ and 30 days of measurements and storage in ambient air (voltage sweep rate $80 \mathrm{mV} \mathrm{s}^{-1}$ ). (b) Extracted on/ off ratios and threshold-voltages for this device after bias stress and storage in air. 
ionogel and the gold top gate are likely to act as gas diffusion barriers and allow for greater air-stability. The ionogel-gated P3HT transistors showed remarkably stable on/off ratios and reversible threshold shifts. Fig. 6a shows the initial transfer characteristics measured in ambient air and after 12 days of storage in air. Additionally, for the first two days the device was stressed by performing transfer measurements every $5 \mathrm{~min}$ for 48 hours. The overall current-voltage characteristics did not change much, but the threshold shifted to more positive values and the on/off-ratio decreased slightly indicating p-doping. After storing the device for 10 days in ambient air the threshold voltage shifted back to its initial value. Further bias stress for two days again led to a positive threshold voltage shift, which likewise was reversible by storage in air for several days (see Fig. 6b).

\section{Flexible ionogel-gated P3HT transistors}

The lightly crosslinked ionogels are quite soft and conformable, which makes them ideal for fabricating flexible transistors on thin polymer substrates such as PET (thickness $50 \mu \mathrm{m}$ ). P3HT was spin-coated from 1,2-dichlorobenzene onto the PET substrates with interdigitated source-drain electrodes. The ionogel was then spray-coated on top from an acetone solution

(a)

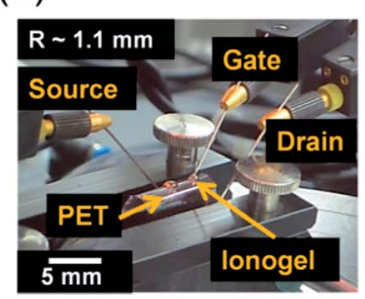

(c)

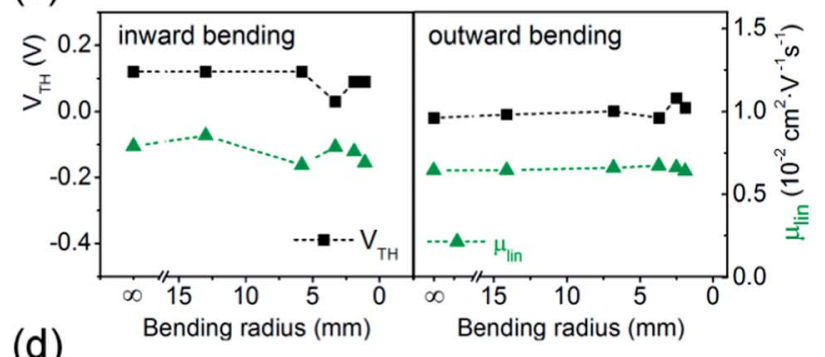

(d)

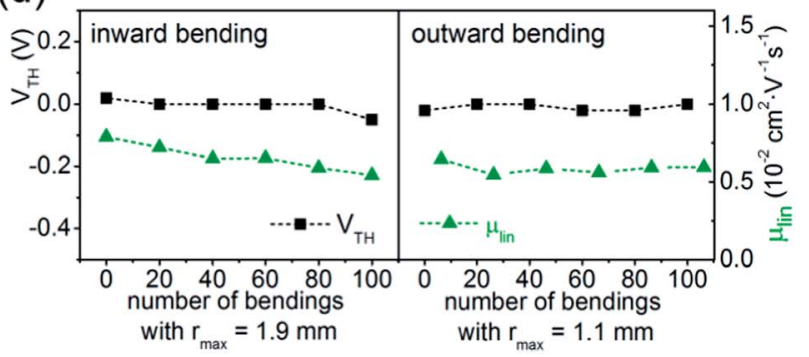

Fig. 7 (a) Optical image of the ionogel-gated P3HT transistor on PET during bending. (b) Transfer characteristics in nitrogen atmosphere for a flat device and for inward and outward bending, voltage sweep rate: $80 \mathrm{mV} \mathrm{s}^{-1}$. Device parameters $\left(V_{\mathrm{TH}}\right.$ and $\left.\mu_{\text {lin }}\right) v s$. bending radius (c) and number of bendings (d). at $60{ }^{\circ} \mathrm{C}$ before a gold gate electrode was evaporated. The device geometry was equivalent to that shown in Fig. 5a. All components were stable under mechanical stress (see Fig. 7a). The P3HT EGTs turned on at $0.45 \mathrm{~V}$ and on/off ratios of $10^{3}$ were obtained (Fig. 7b). The hole mobility was lower $\left(0.008 \mathrm{~cm}^{2} \mathrm{~V}^{-1}\right.$ $\mathrm{s}^{-1}$ ) than on glass probably due to the roughness of the substrate and thus inferior film formation. The performance of the EGTs was stable under bending stress (see Fig. 7c), with stress being applied along the charge transport direction. For inward bending, i.e. compressive stress, the linear field-effect mobility changed only slightly from $0.008 \mathrm{~cm}^{2} \mathrm{~V}^{-1} \mathrm{~s}^{-1}$ to $0.007 \mathrm{~cm}^{2} \mathrm{~V}^{-1} \mathrm{~s}^{-1}$ at a maximum bending radius of $r=1.9 \mathrm{~mm}$. For the outward bending direction, i.e. tensile stress, the device characteristics were stable down to a bending radius of $r=$ $1.1 \mathrm{~mm}$, which corresponds roughly to a strain of $2.3 \%$. The EGTs continued to operate after more than 100 bending cycles for both bending directions while the linear mobility decreased only slightly to $0.006 \mathrm{~cm}^{2} \mathrm{~V}^{-1} \mathrm{~s}^{-1}$ (see Fig. 7d). Note that, none of the components, including the gold electrodes, showed any visible damage after the bending cycles.

\section{Conclusions}

In summary, we synthesized and characterized a new type of silica ionogel, in which the cation of the ionic liquid is involved in the network formation. The obtained positively charged network is soluble in acetone and easily processed from solution by spray-coating at low temperatures. The ionogel shows promising properties for application in flexible electronics, such as flexibility and high capacitance. Importantly, it is possible to evaporate metal gate electrodes on top of thin films of the ionogel. We demonstrated its applicability as a high capacitance electrolyte in low voltage organic FETs and simple circuits, including flexible polymer transistors on PET substrates with remarkably stable performance under mechanical stress and bias stress in ambient air. By decreasing the ionogel film thickness the switching speeds are expected to improve and the devices should be fast enough for simple printed circuit applications.

\section{Acknowledgements}

This research was funded by the Deutsche Forschungsgemeinschaft (DFG) via the Research Training Group 'Disperse Systems for Electronic Applications' (GRK 1161) and the Cluster of Excellence 'Engineering of Advanced Materials' (EXC 315). The authors are grateful to J. Reiser for help with film thickness measurements and TGA analysis, I. Herzer for DSC analysis of the ionogel and M. Drüschler and B. Huber for their help with electrochemical measurements and fruitful discussions.

\section{Notes and references}

1 S.-H. Wang, S.-S. Hou, P.-L. Kuo and H. Teng, ACS Appl. Mater. Interfaces, 2013, 5, 8477-8485.

2 A. Brandt, S. Pohlmann, A. Varzi, A. Balducci and S. Passerini, MRS Bull., 2013, 38, 554-559. 
3 K. Yu Jin, C. Haegeun, H. Chi-Hwan and K. Woong, Nanotechnology, 2012, 23, 065401.

4 S. Saricilar, D. Antiohos, K. Shu, P. G. Whitten, K. Wagner, C. Wang and G. G. Wallace, Electrochem. Commun., 2013, 32, 47-50.

5 A. Lewandowski and A. Świderska, Solid State Ionics, 2003, 161, 243-249.

6 P. Wang, S. M. Zakeeruddin, P. Comte, I. Exnar and M. Grätzel, J. Am. Chem. Soc., 2003, 125, 1166-1167.

7 J. Nei de Freitas, A. F. Nogueira and M.-A. De Paoli, J. Mater. Chem., 2009, 19, 5279-5294.

8 M. Gorlov and L. Kloo, Dalton Trans., 2008, 2655-2666.

9 J. H. Cho, J. Lee, Y. He, B. S. Kim, T. P. Lodge and C. D. Frisbie, Adv. Mater., 2008, 20, 686-690.

10 J. H. Cho, J. Lee, Y. Xia, B. Kim, Y. He, M. J. Renn, T. P. Lodge and C. D. Frisbie, Nat. Mater., 2008, 7, 900-906.

11 Y. Yomogida, J. Pu, H. Shimotani, S. Ono, S. Hotta, Y. Iwasa and T. Takenobu, Adv. Mater., 2012, 24, 4392-4397.

12 K. Hong, S. H. Kim, K. H. Lee and C. D. Frisbie, Adv. Mater., 2013, 25, 3413-3418.

$13 \mathrm{~J} . \mathrm{Pu}, \mathrm{Y}$. Yomogida, K.-K. Liu, L.-J. Li, Y. Iwasa and T. Takenobu, Nano Lett., 2012, 12, 4013-4017.

14 M. Ha, J. W. Seo, P. L. Prabhumirashi, W. Zhang, M. L. Geier, M. J. Renn, C. H. Kim, M. C. Hersam and C. D. Frisbie, Nano Lett., 2013, 13, 954-960.

15 I. Lokteva, S. Thiemann, F. Gannott and J. Zaumseil, Nanoscale, 2013, 5, 4230-4235.

16 S. Dasgupta, R. Kruk, N. Mechau and H. Hahn, ACS Nano, 2011, 5, 9628-9638.

17 J. Le Bideau, L. Viau and A. Vioux, Chem. Soc. Rev., 2011, 40, 907-925.

18 T. P. Lodge, Science, 2008, 321, 50-51.

19 Y. Xia, W. Zhang, M. Ha, J. H. Cho, M. J. Renn, C. H. Kim and C. D. Frisbie, Adv. Funct. Mater., 2010, 20, 587594.

20 S. H. Kim, K. Hong, W. Xie, K. H. Lee, S. Zhang, T. P. Lodge and C. D. Frisbie, Adv. Mater., 2012, 25, 1822-1846.

21 T. Fujimoto and K. Awaga, Phys. Chem. Chem. Phys., 2013, 15, 8983-9006.

22 K. H. Lee, M. S. Kang, S. Zhang, Y. Gu, T. P. Lodge and C. D. Frisbie, Adv. Mater., 2012, 24, 4457-4462.

23 S. Thiemann, S. J. Sachnov, F. Pettersson, R. Bollström, R. Österbacka, P. Wasserscheid and J. Zaumseil, Adv. Funct. Mater., 2014, 24, 625-634.
24 P. Wang, S. M. Zakeeruddin, P. Comte, I. Exnar and M. Grätzel, J. Am. Chem. Soc., 2003, 125, 1166-1167.

25 M.-A. Néouze, J. Le Bideau, F. Leroux and A. Vioux, Chem. Commun., 2005, 1082.

26 M.-A. Néouze, J. Le Bideau and A. Vioux, Prog. Solid State Chem., 2005, 33, 217-222.

27 M.-A. Néouze, J. Le Bideau, P. Gaveau, S. Bellayer and A. Vioux, Chem. Mater., 2006, 18, 3931-3939.

28 J. Le Bideau, P. Gaveau, S. Bellayer, M. A. Néouze and A. Vioux, Phys. Chem. Chem. Phys., 2007, 9, 5419.

29 J. Le Bideau, J.-B. Ducros, P. Soudan and D. Guyomard, Adv. Funct. Mater., 2011, 21, 4073-4078.

30 A. I. Horowitz and M. J. Panzer, J. Mater. Chem., 2012, 22, 16534.

31 S.-M. Lee, H.-J. Yoon, J.-H. Kim, W.-J. Chung and Y.-S. Lee, Pure Appl. Chem., 2007, 79, 1553-1559.

32 M. Freemantle, An Introduction to Ionic Liquids, RSC Publishing, Cambridge, 2010.

33 P. Wasserscheid and T. Welton, Ionic Liquids in Synthesis, Weinheim, 2008.

34 A. Martinelli and L. Nordstierna, Phys. Chem. Chem. Phys., 2012, 14, 13216-13223.

35 J. Kiefer, J. Fries and A. Leipertz, Appl. Spectrosc., 2007, 61, 1306-1311.

36 L. Herlogsson, X. Crispin, N. D. Robinson, M. Sandberg, O. J. Hagel, G. Gustafsson and M. Berggren, Adv. Mater., 2007, 19, 97-101.

37 O. Larsson, E. Said, M. Berggren and X. Crispin, Adv. Funct. Mater., 2009, 19, 3334-3341.

38 A. Malti, M. Berggren and X. Crispin, Appl. Phys. Lett., 2012, 100, 183302.

39 I. Capek, Adv. Colloid Interface Sci., 2004, 112, 1-29.

40 Y. He and T. P. Lodge, Macromolecules, 2007, 41, 167-174.

41 K. H. Lee, S. Zhang, T. P. Lodge and C. D. Frisbie, J. Phys. Chem. B, 2011, 115, 3315-3321.

42 S. Dasgupta, G. Stoesser, N. Schweikert, R. Hahn, S. Dehm, R. Kruk and H. Hahn, Adv. Funct. Mater., 2012, 22, 49094919.

43 H.-H. Liao, C.-M. Yang, C.-C. Liu, S.-F. Horng, H.-F. Meng and J.-T. Shy, J. Appl. Phys., 2008, 103, 104506-104508.

44 W. Xie and C. D. Frisbie, J. Phys. Chem. C, 2011, 115, 1436014368.

45 A. C. Arias, F. Endicott and R. A. Street, Adv. Mater., 2006, 18, 2900-2904. 